\title{
ALiS: Entwicklung einer Designtheorie für Augmented Living Spaces zur erweiterten Autonomie älterer und kognitiv eingeschränkter Menschen
}

\author{
Martin Böhmer (D) · Johannes Damarowsky (D) Sophie Parschat • \\ Victor-Alexander Mahn
}

Eingegangen: 16. Juli 2021 / Angenommen: 1. Dezember 2021 / Online publiziert: 23. Dezember 2021

(C) Der/die Autor(en) 2021

Zusammenfassung Der Alltag älterer und kognitiv eingeschränkter Menschen kann mithilfe von informationstechnologischen Lösungen erheblich unterstützt werden. In diesem Kontext ist, wie unterschiedliche Ansätze bereits zeigen, der Einsatz von Augmented Reality für viele Einsatzszenarien eine vielversprechende Lösung. Jedoch stellen die üblicherweise genutzten Head-Mounted-Displays eine technische Hürde dar und können nicht ausnahmslos von allen Personen getragen werden. Als alternativen Lösungsansatz entwickelt der vorliegende Beitrag einen Augmented Living Space (ALiS). Dieser nutzt anstatt Headsets oder anderen tragbaren Geräten Spatial Augmented Reality, welche bspw. durch Beamer und Lautsprecher implementiert wird. Damit können individuelle, bedürfnisorientierte Funktionen ergonomisch und dennoch immersiv bereitgestellt werden. Hierdurch sollen betroffene Menschen befähigt werden, länger autonom leben zu können, ohne dabei auf tragbare Technik angewiesen zu sein. Ein ALiS gewährt dabei insbesondere eine nutzerzentrierte Unterstützung in den Bereichen der Wahrnehmung, Mobilität, Organisation sowie Medizin und ermöglicht die Herausforderungen des Alltags leichter zu bewältigen. Spatial Augmented Reality ist aktuell ein nur wenig erforschter Ansatz und wird vornehmlich in der Produktion eingesetzt. Daher werden in diesem Beitrag Designanforderungen, Designprinzipien und Design-Features einer Designtheorie formuliert, anhand derer zukünftig ein ALiS für ältere und kognitiv eingeschränkte Menschen mithilfe von Spatial Augmented Reality zielführend gestaltet und eingesetzt werden kann. Zur Definition der nutzerspezifischen und technischen Anforde-

\footnotetext{
Martin Böhmer $(\bowtie) \cdot$ Johannes Damarowsky

Professur für Wirtschaftsinformatik, insb. Betriebliches Informationsmanagement, Institut für Wirtschaftsinformatik und Operations Research, Martin-Luther-Universität Halle-Wittenberg, Universitätsring 3, 06108 Halle (Saale), Deutschland

E-Mail: martin.boehmer@wiwi.uni-halle.de

Sophie Parschat · Victor-Alexander Mahn

DENKUNDMACH Parschat, Mahn GbR, Kleine Klausstraße 14, 06108 Halle (Saale), Deutschland
} 
rungen wurde eine zweistufige strukturierte Literaturanalyse sowie eine moderierte Fokusgruppe mit Expert*innen und Anwender*innen durchgeführt. Die entwickelte Designtheorie besteht aus 3 Designanforderungen, 8 Designprinzipien sowie 13 Design-Features und bildet die Grundlage für die Entwicklung eines ALiS-Prototypen. Die wahrgenommene Nützlichkeit der Designtheorie für die Entwicklung eines ALiS wurde durch eine Befragung von Augmented Reality- und Workflow-ManagementExperten positiv evaluiert.

Schlüsselwörter Spatial Augmented Reality · Augmented Living Space · Design Requirements · Design Principles · Designtheorie · Ambient Assisted Living

\title{
ALiS: Development of a Design Theory for Augmented Living Spaces to Enhance Autonomy of the Elderly and Cognitively Impaired
}

\begin{abstract}
The everyday lives of older and cognitively impaired people can be significantly supported with the help of information technology solutions. In this context, as various approaches have already shown, the use of augmented reality is a promising solution for many application scenarios. However, the commonly used head-mounted displays represent a technical hurdle and cannot be worn by all persons without exception. As an alternative solution, this paper develops an Augmented Living Space (ALiS), that does not use headsets or other wearable devices, but Spatial Augmented Reality, which is implemented by beamers and audio devices. Thus, individual, need-oriented functions can be provided ergonomically and yet immersively. The aim is to enable affected people to live autonomously for longer without having to rely on portable technology. In particular, an ALiS provides user-centered support in the areas of perception, mobility, organization, and medicine, making it easier to cope with the challenges of everyday life. Spatial Augmented Reality is currently a scarcely explored approach and is mainly used in manufacturing as well as maintenance. Therefore, this paper presents design requirements, design principles and design features of a design theory that acts as a foundation to develop an ALiS for elderly and cognitively impaired people which can be used purposefully with the help of Spatial Augmented Reality. To define the user-specific and technical requirements, a two-stage structured literature review and a moderated focus group with experts were conducted. The developed design theory consists of 3 design requirements, 8 design principles as well as 13 design features and forms the basis for the development of an ALiS prototype. Furthermore, the perceived usefulness of the design theory for the development of an ALiS was positively evaluated through a survey of researchers and augmented reality experts.
\end{abstract}

Keywords Spatial Augmented Reality · Augmented Living Space · Design Requirements · Design Principles · Design Theory · Ambient Assisted Living 


\section{Einleitung}

Im Allgemeinen werden kognitive Einschränkungen als Einbußen der geistigen Leistungsfähigkeit, folglich Störungen in der Wahrnehmung, im Erkennen, im Erinnern, im Denken und im Urteilen wahrgenommen (Bartsch und von Arnim 2019). Kognitive Einschränkungen sind dabei nicht gleichbedeutend mit Demenz und deren schweren/dysfunktionalen Folgen, jedoch stellen sie häufig ein hohes Risiko hin zu einer demenziellen Entwicklung dar (Gauthier et al. 2006). Bartsch und von Arnim (2019) sowie Gauthier et al. (2006) führen aus, dass Menschen mit milden kognitiven Einschränkungen, bei denen noch keine Demenz-Diagnose gestellt wurde, auch weiter autonom ihren Alltag bewältigen können, dabei jedoch EinbuBe an kognitiver Performanz hinnehmen müssen, die Prozesse im Alltag (Kochen, Anziehen, etc.) beeinflussen können. Heutzutage bieten informationstechnologische Lösungen eine Vielzahl an Möglichkeiten, um das Leben von älteren und kognitiv eingeschränkten Menschen zu unterstützen. Aufgrund der ansteigenden Zahl von älteren Menschen, nehmen in der Praxis ebenfalls die digitalen Lösungsansätze eine immer entscheidendere Rolle ein (Preum et al. 2021). Ein aktuell diskutierter Ansatz hierfür ist speziell die Anwendung von Augmented Reality (AR), um kognitiv eingeschränkten Personen dabei zu helfen, den Alltag und damit verbundene Aufgaben zu bewältigen. Dabei kommen in der Praxis primär Head-Mounted-Displays (HMD) zum Einsatz, welche die Grenzen der realen und virtuellen Welt miteinander verschmelzen lassen. Neben der meist geringen Akkukapazität, den komplexen Menüs oder des verringerten Sichtfeldes, können HMDs speziell von Personen mit kognitiven Einschränkungen aufgrund körperlicher und medizinischer Voraussetzungen nicht ausnahmslos getragen werden. Einen alternativen Ansatz zur AR-basierten Unterstützung kann Spatial Augmented Reality (SAR) bieten, welche Informationen, Objekte, etc. mithilfe von visuellen Projektionen und auditiver Kommunikation darstellt (Raskar et al. 1998). Dabei ist ferner kein zusätzliches tragbares Gerät notwendig, da relevante Informationen direkt in das Umfeld der Nutzer*innen projiziert werden.

Die Anwendung von Spatial Augmented Reality ermöglicht es, die kognitive Last der Anwender*innen zu reduzieren und erhöht gleichzeitig deren Leistungsfähigkeit hinsichtlich alltäglicher Aufgaben (Baumeister et al. 2017). Jedoch sind Lösungsansätze für die Anwendung von SAR in der Pflege kaum existent, da sich die Anwendung in der Praxis vorrangig auf die industrielle Produktion und Wartung konzentriert, wobei erhebliche Produktivitätsanstiege bei gleichzeitiger Fehlerminimierung erreicht werden (Rupprecht et al. 2020; Cardoso et al. 2020; Tavares et al. 2019). Vereinzelte Anwendungen spatialer AR im Kontext kognitiver Einschränkungen beschäftigen sich mit projizierenden Robotern, die Erinnerungen visualisieren (Yang et al. 2018) oder in Smart-Home-Umgebungen Benachrichtigungen projizieren (Wegerich et al. 2010). Diese Ansätze können jedoch die Pflege und den Erhalt der Selbstständigkeit von älteren sowie kognitiv eingeschränkten Personen nicht vollumfänglich adressieren. Eine wesentliche Einschränkung dieser Ansätze stellt dabei die geringe Flexibilität und Möglichkeit zur Adressierung komplexer Abläufe dar. Denn entsprechende Systeme sind nicht in der Lage, auf sich dynamisch ergebende Situationen zu reagieren, komplexe Kontextinformationen zu verarbeiten 
und somit betroffene Personen adäquat und individuell zu unterstützen. Ein Ansatz, um dies zu ermöglichen ist die Verbindung von Spatial Augmented Reality mit kontextabhängigen Sensoren im alltäglichen Lebensumfeld der Nutzer*innen. Hierbei ermöglicht der aktuelle state-of-the-art der Sensortechnologie, auch hinsichtlich tragbarer Sensoren, eine fundierte Basis für Echtzeit-Datenübermittlung, die für eine Anwendung in der Pflege prädestiniert ist (Baig et al. 2019; Mshali et al. 2018). Es existiert keine Designtheorie, welche die eben genannten Charakteristika ganzheitlich auffasst und anhand derer ein Augmented Living Space (ALiS) entwickelt werden kann. Designtheorien können zur systematischen Unterstützung von IT-System-Designer*innen und Entwickler*innen genutzt werden, welche die Designanforderungen an das IT-System umfassen und die damit verbundenen Designprinzipien adressieren (Baskerville und Pries-Heje 2010). Da keine Designtheorie für einen ALiS existiert und um somit IT-System-Designer*innen und Entwickler*innen sowie letztlich kognitiv eingeschränkte Personen zu unterstützen, ist die Forschungsfrage für diesen Beitrag wie folgt formuliert:

RQ: Was sind die Designanforderungen und Designprinzipien einer Designtheorie für Assistenzsysteme für kognitiv eingeschränkte Personen auf Basis spatialer Augmented Reality?

Um die Forschungsfrage zu beantworten, stellt dieser Beitrag einen Augmented Living Space für kognitiv beeinträchtigte Personen vor, welcher mithilfe von spatialer AR sowie Sensoren in Echtzeit die Umgebungsbedingungen von Nutzer*innen wahrnimmt und somit kontextabhängige Benachrichtigungen visuell projizieren oder auditiv kommunizieren kann. Hierdurch sollen entsprechende Personen im Alltag zu mehr und insbesondere längerer Autonomie befähigt werden, indem sie sich selbständiger in ihrer vertrauten Umgebung bewegen können. ALiS soll ältere Personen sowie Nutzer*innen mit milden kognitiven Einschränkungen in ihrer Wahrnehmung, Mobilität, Organisation und medizinischen Pflege unterstützen, wobei exemplarisch Gefahrenmeldungen oder die Navigation in der Wohnung ermöglicht wird, ohne dass Personen auf tragbare Technologien angewiesen sind. Der vorliegende Beitrag erweitert simultan das Forschungsfeld von SAR und digitalen Assistenztechnologien, indem nutzerspezifische sowie technische Anforderungen als Design Requirements (DR) für einen ALiS definiert werden. Zudem werden Design Principles (DP) als allgemeine Lösungsansätze für diese Design Requirements identifiziert. Um insbesondere eine praxisnahe Konzeption zu erreichen, sodass Entwickler*innen, Pflegekräfte und kognitiv eingeschränkte Personen davon in ihrem Alltag profitieren können, entwickelt dieser Beitrag ebenfalls Design Features (DF) für eine konkrete Implementierung eines ALiS-Prototypen. Somit wird die Realisierbarkeit sowie Sinnhaftigkeit der Design Principles und Features validiert.

\section{Forschungsmethodik}

Um eine größtmögliche wissenschaftliche Stringenz zu gewährleisten und den Strukturansatz für einen ALiS zu realisieren, wurde die Design Science Research-Methodik nach Vaishnavi und Kuechler (2015) angewandt, welche die folgenden fünf 


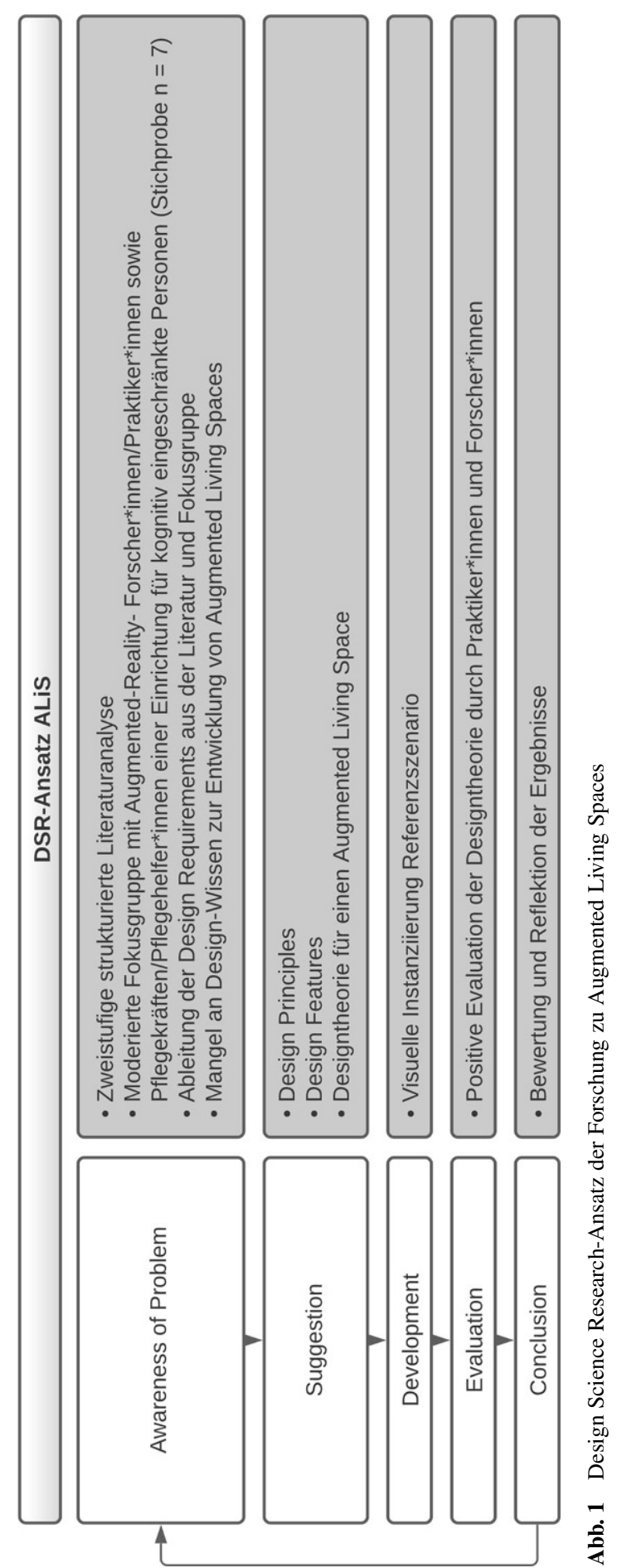


Schritte umfasst: Problemidentifikation, Empfehlung, Entwicklung, Evaluation, und Schlussfolgerung. Die DSR-Methodik ist dabei ein valider Ansatz, um Anforderungen und Vorschläge hinsichtlich Informationssystemen in der digitalen Pflege zu konzipieren (Miah et al. 2017). Der vorliegende Beitrag stellt dabei einen abgeschlossenen DSR-Zyklus vor, dessen Ergebnis eine evaluierte Designtheorie ist, die von Praktiker*innen adaptiert werden kann. In diesem Beitrag werden die Design Requirements, Design Principles und Design Features eingehender thematisiert und vorgestellt, da sie das Fundament einer Designtheorie darstellen und zur Lösung des beschriebenen Problems beitragen (Baskerville und Pries-Heje 2010). Die DRs beschreiben hierbei die prinzipiellen Zielsetzungen und stellen gleichsam Meta-Anforderungen für den späteren Prototypen dar (Baskerville und Pries-Heje 2010; Walls et al. 1992). Die DPs des ALiS sind im entsprechenden Kontext vorschreibend und allgemeingültig, indem sie festlegen, wie ein allgemeingültiger Prototyp konzipiert werden sollte, um den Design Requirements hinsichtlich der Zielgruppe gerecht zu werden (Fu et al. 2016). Dabei wurden die DPs nach Möller et al. (2020) durch einen Supportive Approach abgeleitet, dessen a priori Festlegung eine präskriptive Formulierung (Fu et al. 2016) nahe legt. Die Design Features beziehen sich in diesem Beitrag hingegen auf die speziellen Features unserer Prototyp-Entwicklung und beschreiben die konkrete Ausführung der Design Principles. (Abb. 1).

\section{Problemidentifikation}

Im zugrundeliegenden Design Science Research-Ansatz wurde eine zweistufige strukturierte Literaturanalyse (SLR) nach vom Brocke et al. (2009) durchgeführt, deren Charakteristika nach der Taxonomie von Cooper (1998) Tab. 1 entnommen werden können. Nach den ersten Datenbanktreffern wurden die Beiträge zuerst nach Titel und Abstract gefiltert und ausgewählt. Anschließend wurde der Volltext der

Tab. 1 Charakteristika der zweistufigen strukturierten Literaturanalyse

\begin{tabular}{|c|c|c|}
\hline & SLR 1 & SLR 2 \\
\hline Suchterm & $\begin{array}{l}\text { ("spatial augmented reality" OR "spatial } \\
\text { mixed reality") AND ("architect*" OR } \\
\text { "ontology" OR "design") }\end{array}$ & $\begin{array}{l}\text { ("digital support“ OR "digital assist*") } \\
\text { AND "elderly“ OR ("cognitive“ AND } \\
\text { (,impairment“ OR ,guidance“)) }\end{array}$ \\
\hline Suchfelder & Titel, Abstract & Titel, Abstract \\
\hline Datenbanken & \multicolumn{2}{|c|}{$\begin{array}{l}\text { ScienceDirect, ACM Digital Library, AIS electronic Library, SpringerLink, IEEE Xplore, } \\
\text { Web of Science, EBSCOhost (Business Source Premier/Academic Search Premier), } \\
\text { JSTOR, PubMed }\end{array}$} \\
\hline$O / T A / F / B F / T R$ & $442 / 147 / 65 / 80 / 7$ & $6.353 / 241 / 38 / 21 / 17$ \\
\hline Fokus & Ergebnisse, Theorie, Anwendungen & Ergebnisse, Anwendungen \\
\hline Zielgruppe & Allgemeine \& spezifische Fachleute & Allgemeine \& spezifische Fachleute \\
\hline Umfang & Repräsentativ & Repräsentativ \\
\hline Ziel & Synthetisieren & \\
\hline Organisation & Konzeptionell & \\
\hline Blickwinkel & Neutrale Wiedergabe & \\
\hline
\end{tabular}

Legende: $O$ Originale Treffer, $T A$ Treffer nach Titel \& Abstract Filter, $F$ Treffer nach Volltext Filter, $B F$ Treffer nach Vorwärts- \& Rückwärtssuche, $T R$ Top Relevanz 
relevanten Treffer analysiert und eine Vorwärts- bzw. Rückwärtssuche der bedeutsamen Treffer durchgeführt. Um state-of-the-art Ergebnisse der involvierten Technologien sicherzustellen, wurde einerseits eine strukturierte Literaturrecherche zu aktuellen Architekturmodellen und Techniken von SAR, andererseits zu neoterischen digitalen Assistenzsystemen in der Pflege durchgeführt. Ziel dessen war es, mögliche technische und konzeptuelle Modellarchitekturen zu identifizieren, welche die Essenz bestehender Anwendungen erfassen und als Leitfaden für die Entwicklung eines Prototypen sowie zur Entwicklung nutzerzentrierter Anforderungen dienlich sein könnten.

In SLR 1 umfasste die Suche mögliche technische und praktische Anwendungsarchitekturen von Spatial Augmented Reality, wobei nach der Vorwärts- und Rückwärtssuche 7 Artikel mit Top-Relevanz verblieben, die potenziell für den Einsatz in einem ALiS relevant erscheinen. Dabei definiert sich die Relevanz hierfür über mögliche SAR Setup-Techniken, erfolgreiche praktische Umsetzungen und nützliche Methoden zur Wahrnehmungsbeeinflussung durch Projektionen. Hinsichtlich der Setup-Techniken ist vor allem die Kompensation eines projizierten Bildes auf einen bestimmten Untergrund von Relevanz, um die Genauigkeit und die Dimensionen des Originalbildes zu wahren. Hierfür stellten Huang et al. (2021) eine Methode vor, welche die geometrische und photometrische Kompensation mithilfe eines Deep Neural Networks löst und somit eine (fast) fehlerfreie Darstellung ermöglicht. Ro et al. (2019) nutzen ebenfalls Deep Learning, um auf Basis der Position des Nutzers optimale Projektionsflächen zu identifizieren. Zudem erscheint die Arbeit von Wilson et al. (2012) im Kontext eines ALiS als relevant, da die Autoren eine Methode vorstellen, um Objekte durch eine motorisierte Plattform im ganzen Raum zu projizieren. Erfolgreiche Praxisanwendungen wurden primär durch einen digitalen SARKochassistenten für kognitiv eingeschränkte Personen (Kosch et al. 2019) oder spielerisch interaktive Boden-Projektion zu Inklusionszwecken (Graf et al. 2019) realisiert. Hinsichtlich der Wahrnehmungsbeeinflussung sind vordergründig die Arbeiten von Miyamoto et al. (2018) maßgeblich, die irrelevante Objekte durch Verschieben von Pixeln unscharf erscheinen lassen, sowie Lindlbauer et al. (2017), welche die Wahrnehmung von Objekten beeinflussen, indem deren Umgebung modifiziert wird. SLR 2 versuchte anschließend digitale Assistenzsysteme in der Praxis zu identifizieren, um Leitlinien, Erfolgs-, Risiko- und Klassifizierungsfaktoren zu identifizieren, wobei nach Vorwärts- und Rückwärtssuche 17 Artikel mit Top-Relevanz gefunden werden konnten. Entsprechende Systeme dienen derweil als Referenz und Vergleich für die Erfüllung der später vorgestellten Design Requirements. Dabei erscheint die Arbeit von Morris (1994) als Basis für die digitale Gestaltung der Projektionen zu dienen, da hierbei Empfehlungen der Darstellung für kognitiv eingeschränkte Personen artikuliert werden. Die Arbeit von Lockerbie und Maiden (2019) beschreibt andererseits, inwiefern digitale Assistenzsysteme in der Pflege zu einer Steigerung der Lebensqualität kognitiv beeinträchtigter Personen führen können und wie diese konzipiert sein sollten. Chang et al. (2014) zeigen anschaulich, wie kognitiv eingeschränkte Personen mittels klassischer AR spielerisch dazu befähigt werden, ihre Selbständigkeit zu erhöhen. Ein Anwendungssystem, welches spatiale AR als digitale Assistenz nutzt, wurde von Yang et al. (2018) vorgestellt, wobei ein Roboter Erinnerungen an verschiedene Flächen projiziert. Zudem stellen Luis-Ferreira 
et al. (2019) aktuelle Sensorik-Ansätze für digitale Assistenzsysteme und deren Anwendung bei kognitiv eingeschränkten Personen vor, wohingegen Goonawardene et al. (2018) aktuelle Sensortechnologie in ein Assistenzsystem für ältere Personen einbetten. Überdies gibt es eine Vielzahl an digitalen Assistenzsystemen für ältere Personen, die spezifische Aspekte des alltäglichen Lebens (Monitoring, Socializing, Caregiving, etc.) unterstützen und den Begriff des Ambient Assisted Living (AAL) prägen (Tablado et al. 2005; Seet et al. 2016; Park et al. 2017; Lema-Sarmiento et al. 2020; Benavides et al. 2020; Spoladore et al. 2021). Des Weiteren wurden durch Tazari et al. (2010) konzeptionelle sowie durch Antonino et al. (2011) technische Referenzarchitekturen für digitale Assistenzsysteme vorgestellt, wobei der Beitrag von Garces et al. (2020) die relevantesten Referenzarchitekturen für AAL analysiert und hiermit die maßgebendsten Anforderungen bündelt. Dabei zeigen Garces et al. (2020) sowie Memon et al. (2014), dass einerseits die Qualität und Vollständigkeit von digitalen Assistenzsystemen und AAL-Systemen über den Lauf der Zeit abnimmt und andererseits aktuelle Systeme die essenziellen Aspekte nicht ganzheitlich berücksichtigen. In Hinblick auf die Klassifizierung digitaler Assistenzsysteme stellen Knote et al. (2019) ein Framework bereit, anhand dessen sich Systeme konzeptuell kategorisieren lassen, wodurch Anforderungen, Prinzipien, Unterschiede und Gemeinsamkeiten vergleichbar werden.

Weitere Ansätze und zusätzliche Literatur werden nachfolgend in Kap. 4 diskutiert. Ein ganzheitlicher, dynamischer und modularer Ansatz zur Unterstützung ältere und kognitiv eingeschränkter Personen ist dabei in der Literatur (Garces et al. 2020; Memon et al. 2014) nicht existent und wird daher mit einem ALiS aufgegriffen.

\section{Verwandte Arbeiten}

Das Fundament dieser Arbeit und die damit verbundene Konzipierung eines ALiS bildet das Grundlagenwerk von Raskar et al. (1998) zu SAR. Hierbei wurde erstmals der Begriff Spatial Augmented Reality definiert, wobei Vorteile dieser Technologie erörtert und eine mögliche technische Umsetzung diskutiert wurden. Speziell wurden Oberflächenformextraktionen, Rendering-Methoden und Erfassungsartefakte vorgestellt, die von nachfolgenden Beiträgen in diesem Forschungsfeld aufgenommen und durch SLR 1 identifiziert wurden. Neben den genannten Beiträgen in Kap. 3 ist dabei in einer nachfolgenden Vorwärtssuche von SLR 1 der Beitrag von Grundhöfer und Iwai (2018) lokalisiert wurden, welcher aktuelle state-of-the-art Algorithmen, Hardware und Anwendungen des in SAR verwendeten Projektions-Mapping bündelt und somit ebenfalls Relevanz für die technische Realisierung eines ALiS aufweist. Hinsichtlich der technischen Umsetzung ist zudem der Beitrag von Di Donato et al. (2015) bedeutsam, die verschiedene Oberflächenprojektionen erforschten und somit die adäquatesten Oberflächen für SAR feststellen konnten. Ebenfalls maßgeblich sind in diesem Kontext Arbeiten, die eine Projektion, unter anderem mit Hilfe Künstlicher Intelligenz, auf sich bewegende Objekte ermöglichen (Gomes et al. 2021; Kobayashi und Hashimoto 2014; Lee et al. 2019), wobei überdies verfolgende Projektionen auf fliegende Drohnen realisiert werden können (Darbar et al. 2019). Diese Arbeiten dienen als Referenz für die technische Konzipierung eines ALiS und 
stellen daher wichtige verwandte Beiträge zu dieser Thematik dar. Hinsichtlich der nutzerspezifischen Anforderungen und zusätzlich zu den genannten Ergebnissen aus SLR 2 sind aus Sicht der digitalen Assistenzsysteme ebenfalls die Arbeiten von Baig et al. (2019) relevant, die eine systematische Analyse von tragbaren Sensoren im IoT-Kontext für pflegebedürftige Menschen durchgeführt haben, sowie Varriale et al. (2020), welche den aktuellen Stand der Forschung hinsichtlich Automatisierungstechnologien für Personen mit Beeinträchtigungen vorstellen. Zudem bieten die Arbeiten von Fernando et al. (2016) Grundlagen und Anforderungen für assistiertes Leben und Smart-Home-Architekturen, wohingegen Block et al. (2020) Interviews mit Pflegepersonen von kognitiv eingeschränkten Menschen durchführte, um die Nutzung und Akzeptanz von digitalen Assistenzsystemen zu eruieren. In diesem Zusammenhang wurden ebenfalls Anforderungen an passende Technologien im Pflegekontext aus Sicht der Pflegekräfte erhoben, wobei betroffene Personen digitalen Assistenzsystemen gegenüber aufgeschlossen sind und diese als Unterstützung empfinden (Saborowski und Kollak 2015). Ferner zeigt der Beitrag von Kosch et al. (2019), inwieweit Design Requirements und Design Principles für eine SmartKitchen Umgebung für kognitiv eingeschränkte Personen formuliert und inwiefern daraus Design-Implikationen abgeleitet werden können. Der vorliegende Beitrag unterscheidet sich dahingehend von den anderen Arbeiten, dass SAR als digitales Assistenzsystem für kognitiv eingeschränkte Personen fungiert, indem Echtzeitdaten per Kamera oder Sensorik, bspw. ein heißer Herd, ausgewertet und die projizierten Benachrichtigungen darauf ausgerichtet werden. Dies ermöglicht im Gegensatz zu den genannten Beiträgen eine neue Ebene der Flexibilität und ermöglicht die Reaktion auf Geschehnisse in Echtzeit. In Hinblick auf digitale Assistenzsysteme für ältere Personen ist zudem die ethische Betrachtung von großer Relevanz und wird durch den Beitrag von Mueller und Heger (2018) adressiert. Die Autoren haben dabei grundlegende Werte in konkrete Normen übersetzt und somit spezifische Designanforderungen für ein digitales Assistenzsystem der Physiotherapie formuliert, anhand derer mögliche Konfliktpotentiale entschärft werden können.

\section{Moderierte Fokusgruppe}

Aufbauend auf der zweistufigen Literaturanalyse zur Problemidentifikation wurde überdies eine moderierte Fokusgruppe (MFG) durchgeführt, deren Ziel es war, stringente Design Requirements abzuleiten und in einer Designtheorie zu etablieren. Nach Morgan (1997) ist diese qualitative Forschungsmethode besonders für umfangreiche Einblicke in einen Sachverhalt und die Prototypen-Entwicklung prädestiniert. Dabei führt ein Moderator die Gruppendiskussion, welche insbesondere durch die Interaktion zwischen den Teilnehmer*innen lebt und somit immer neue Blickwinkel generiert (Morgan 1997). Hierfür bestand die teilnehmende Gruppe aus $n=7$ Teilnehmer*innen, einschließlich fünf Pflegekräften (eine Pflegeleitung und vier Pflegefachkräfte), die mehrjährige Erfahrung im Umgang mit älteren und kognitiv eingeschränkten Personen vorweisen, und zwei AR-Praktiker*innen, welche ihrerseits mehrjährige Erfahrung auf dem Gebiet der Augmented sowie Virtual Reality vorweisen können. Das Vorgehen für die MFG umfasste fünf Schritte: 
1) Bekräftigen des Forschungsthemas, 2) Diskutieren der Forschungs-Problematik, 3) Protokollieren, 4) Qualitative Inhaltsanalyse der Protokolle, 5) Clustering der Design Requirements. Das Forschungsziel der MFG war es, die unterschiedlichen Ausprägungen der kognitiven Einschränkungen einschätzen zu können und darauf aufbauend nutzerspezifische Anforderungen zu erheben. Dabei fiel die Entscheidung gegen eine direkte Durchführung mit der Zielgruppe der älteren und kognitiv eingeschränkten Personen, da diese meist verminderte und verzerrte Einschätzungen hinsichtlich ihres Alltags mit kognitiven Einschränkungen aufweisen (Chung und Man 2009). Die begleitenden Pflegekräfte hingegen haben eine unvoreingenommene Sicht auf die Bedürfnisse, Handlungen und Herausforderungen der betroffenen Personen und ermöglichen somit eine valide sowie adäquate Datengrundlage.

Um die transkribierten Protokolle kontextuell hinreichend auszuwerten, wurde die Methode der Qualitativen Inhaltsanalyse nach Mayring und Fenzl (2019) gewählt. Das zugrunde liegende inhaltsanalytische Kommunikationsmodell (Mayring und Fenzl 2019) impliziert für die Richtung der Analyse, durch den Text Aussagen über den emotionalen, kognitiven und handlungsorientierten Hintergrund der Proband*innen zu generieren. Dabei wurden induktive Kategorien als zentrales Instrument der Analyse gebildet, welche wiederum Sub-Kategorien beinhalten. Dementsprechende Kategorien lassen sich folgendermaßen formulieren: K1: Alltag von Pflegebedürftigen, K2: Herausforderungen mit kognitiv eingeschränkten Personen, K3: Entwicklung kognitiv eingeschränkter Personen, K4: Kognitive und körperliche Verfassung. Ein wichtiger Aspekt der moderierten Fokusgruppe war es, einen tiefgehenden Einblick in den Alltag kognitiv eingeschränkter Personen zu bekommen (K1). Hierbei wurde durch die Teilnehmer*innen besonders die Struktur des Alltags hervorgehoben und als maßgebend relevant erachtet. Dies umfasst alltägliche Prozesse, wobei der Fokus hauptsächlich auf den Mahlzeiten liegt. Die Alltagsstruktur der kognitiv eingeschränkten Personen wird dabei maßgeblich durch das Individuum selbst vorgegeben, wobei Eckpunkte (bspw. Mahlzeiten, Beschäftigung, Hygiene) weitgehend universal sind. Ferner benötigen betroffene Personen konkrete Ankerpunkte in Form von Gegenständen, Aufgaben oder Abläufen anhand derer sie ihren Alltag bewältigen. Des Weiteren waren die Herausforderungen im Umgang mit kognitiv eingeschränkten Personen ein großer Themenkomplex des Gespräches (K2). Dabei konnten zusammenfassend die Sub-Kategorien Ansprache \& Kommunikation, Wohnsituation, Digitale \& Haptische Signale sowie Anwendungsszenarien gebildet werden. Die Proband*innen empfahlen hierbei einstimmig eine ruhige, eindeutige und prägnante Ansprache/Kommunikation von/mit kognitiv eingeschränkten Personen, da die individuelle Reaktion auf auditive oder visuelle Reize meist differiert. Zudem müsse es auf das jeweilige Individuum und dessen Verfassung angepasst werden, ob möglichst auditive oder visuelle Ansprachen gewählt werden. Die direkte und indirekte Erfassung der Anwendungsszenarien ermöglichte eine Gliederung in angeleitete Tätigkeiten (Medizin, Beschäftigung, Haushalt, Therapie) und erinnerndelaktivierende Tätigkeiten (Kognitive Aktivierung, Alltagsstruktur, Erinnerungen). Entscheidend erschienen ebenfalls die praxisnahen Ausführungen hinsichtlich der Entwicklung kognitiv eingeschränkter Personen (K3). Dabei wurde erfahrungsgemäß berichtet, dass die kognitive Entwicklung ebendieser Personen häufig rückläufig ist und kognitiv eingeschränkte Personen versuchen, Herausforderungen zu 
umgehen. Gleichwohl kann die kognitive Regression durch gezieltes Training zumindest subjektiv gebremst werden, was mit den Aussagen von Bartsch und von Arnim (2019) korrespondiert, jedoch mit der Möglichkeit einer kognitiven Revitalisierung durch die neuronale Plastizität im hohen Alter. Weiterhin entwickelte sich durch die Gruppendiskussion der Themenkomplex der kognitiven und körperlichen Verfassung von Personen mit kognitiven Einschränkungen (K4). Die Proband*innen berichteten hierbei insbesondere über sprunghafte Zustandsänderungen (physisch und kognitiv) und die fehlende Fähigkeit, das „Unvorhersehbare vorauszusehen“. Zudem ist in der Praxis eine kategorische Trennung der kognitiven und körperlichen Zustände (bspw. anhand von Graden) nicht möglich, da die kognitiven Einschränkungen höchst individuell sind und anhand der Gesamtverfassung des Individuums bewertet werden müssen.

Die Fokusgruppe zeigte dabei, dass kognitiv eingeschränkte Personen vor allem eine feste Struktur in ihrem Alltag benötigen und sich bestimmte Ankerpunkte in ihrem Umfeld suchen. Dabei ist besonders eine einfache und eindeutige Kommunikation essenziell, ebenso wie die konstante Aktivierung der kognitiven Fähigkeiten. Überdies ist der Faktor Individualität von großer Relevanz, da kognitive Einschränkungen nicht standardisiert, wohl jedoch modular kategorisiert werden können. In Hinblick auf die digitale Unterstützung von kognitiv eingeschränkten Personen lassen sich durch das Synthetisieren der Kategorien eindeutige nutzerspezifische und technische Anforderungen ableiten, die nun im nachfolgenden Kapitel anhand der Designtheorie erläutert werden.

\section{Designtheorie eines ALiS}

Für die Entwicklung der Designtheorie orientierte sich das methodische Vorgehen an Möller et al. (2020), wobei durch das Definieren von Design Principles das DesignWissen von einer Anwendung hin zu mehreren Anwendungsszenarien transferiert werden kann. Dies ermöglicht ein intuitives Anpassen des Systems in verschiedenen Szenarien ohne dadurch die Design Requirements und Design Principles neu definieren zu müssen.

Die Auswertung der strukturierten Literaturrecherche sowie der Fokusgruppe mit Qualitativer Inhaltsanalyse ermöglichte es, drei essenzielle Design Requirements eines ALiS zu clustern. Diese wiederum unterteilen sich in acht Design Principles, welche ihrerseits insgesamt 13 Design Features enthalten. Dabei illustrieren die Design Requirements die Ziele, welche alle zielgruppen-spezifischen ALiS-Systeme adressieren sollten und stellen hierdurch allgemeingültige Rahmenbedingungen dar (Möller et al. 2020). Weiterhin definieren die Design Principles, welche Lösungsansätze von allen ALiS-Systemen implementiert werden sollten, wohingegen die Design Features speziell die Implementierung von Lösungsansätzen eines ALiS im Kontext der Zielgruppe, Hardware, Software, den Mitarbeiter*innen und Kompetenzen spezifizieren. Diese können somit als Features unseres eigenen Prototypen angesehen werden. Während sich der Design Science Research-Ansatz ferner an der ISO 9241-210:2020 und somit der menschzentrierten Gestaltung interaktiver Systeme orientiert, konnte die Anforderung der Gebrauchstauglichkeit nach ISO 9241- 
11:2018 (2018) in die folgenden drei Kernkomponenten aufgegliedert werden: Zufriedenheit der Benutzer*innen (DR1), Effizienz (DR2) und Effektivität (DR3). DR1 sollte intuitiv verständlich sein und die Anforderung stellen, dass Nutzer*innen einen ALiS mindestens genauso gern oder lieber als ein Alternativsystem (siehe SLR 2) nutzen. Darüber hinaus bedarf es jedoch einer klaren und verständlichen Trennung zwischen Effizienz und Effektivität. Beide Begrifflichkeiten sollten hierbei nicht ökonomisch, sondern aufgabenorientiert betrachtet werden. DR2 beschreibt somit die Anforderung, dass ein ALiS einerseits mit geringem Aufwand von Nutzer*innen bedient werden kann, andererseits aber auch durch Administrator*innen leicht angepasst werden kann. Dabei soll ein ALiS eine mindestens genauso hohe oder höhere Effizienz bei der Nutzung als Alternativsysteme sicherstellen. DR3 beschreibt durch die Effektivität das Bereitstellen aller nützlichen Funktionen zur Erhaltung/ Erweiterung der Autonomie für die Zielgruppe. Mit anderen Worten impliziert dies, dass für eine Zielgruppe unwirksame Funktionen obsolet werden. (Abb. 2).

DP1 adressiert DR1 und definiert die individuell bedürfnisorientierte, intuitive und zielgruppengerechte Interaktion, wodurch die Anforderungen der Individualität aus der Fokusgruppe sowie die nutzerzentrierte Ausrichtung aus der Literatur (Blackler et al. 2020; Graf et al. 2019; Morris 1994; Mueller und Heger 2018) erfüllt werden. Hiermit sollen die kognitiven Einschränkungen berücksichtigt und ein für die Nutzer*innen positives Interaktionserlebnis sichergestellt werden, was in unserem speziellen ALiS-Anwendungskontext für DP1 durch DF 1-6 erreicht wird. Dies umfasst die durch die Fokusgruppe geforderte einfache und eindeutige Sprache (DF1), das Nutzen von Metaphern statt Abstraktionen, um nicht die reale Welt zu digitalisieren, sondern virtuelle Hinweise in die reale Welt $\mathrm{zu}$ integrieren (Blackler und Popovic 2016) (DF2), sowie Mehrfachkodierungen hinsichtlich Ton, Schrift und Sprache (Kosch et al. 2019; Morris 1994; Picking et al. 2012) (DF3). Letzteres ermöglicht es bspw. intuitive Farbkodierungen darzustellen (rot für Wärme oder blau für Kälte). Ferner sollte ALiS eine niedrigschwellige Interaktion beinhalten, wodurch auch Menschen mit sporadischer Technologie-Erfahrung das System intuitiv nutzen können (Blackler et al. 2020; Garces et al. 2020; Tazari et al. 2010) (DF4). Zudem sollten synthetische Inhalte in einer Art Ankerfunktion (siehe Abb. 3) an physischen Objekten fixiert werden, um eine Gleichmäßigkeit und einen Erinnerungsbezug herzustellen (DF5), was durch die Fokusgruppe als äußerst relevant erachtet wurde. Gleichzeitig erscheint eine nutzerzentrierte, agil-iterative Entwicklung nach ISO 9241-210:2019 sinnvoll, um das System bestmöglich auf die Zielgruppe ausrichten zu können (DF6).

Wenngleich sich DP2 ähnlich zu DP1 liest, so zielt es jedoch nicht auf die Interaktion, sondern auf die Kommunikation ab, da die qualitative Auswertung der MFG einen Schwerpunkt auf die individuelle Kommunikation erschließen ließ und jenes Prinzip in der Literatur als relevant erachtet wird (Kosch et al. 2019; Morris 1994). Überdies sollten hier ebenfalls die kognitiven Einschränkungen berücksichtigt werden, um das Kommunikationserlebnis zu intensivieren. DP2 beinhaltet dabei ebenfalls DF3, DF5 sowie DF6. Zugleich wird es um DF7 erweitert, womit die Funktion des Eye- oder-Body-Tracking gefordert wird, sodass die besagten Ankerpunkte indirekt/direkt angesteuert und Informationen blickrichtungs-basiert hervorgehoben werden können. 


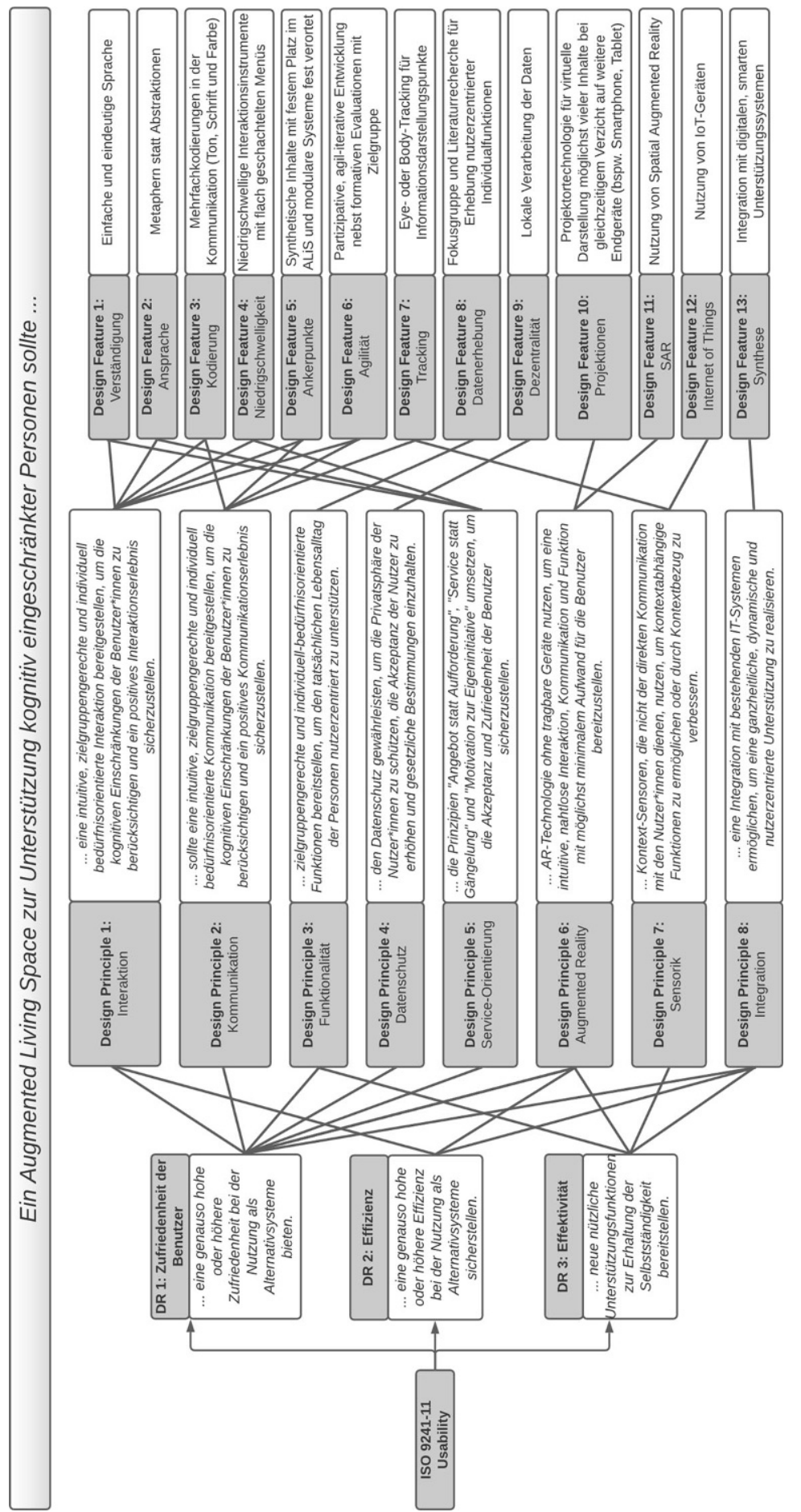

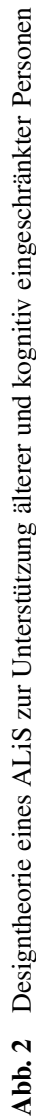


DP3 definiert überdies die gleichzeitige Umsetzung von DR1 und DR3 und die aus der Fokusgruppe und Literatur (Antonino et al. 2011; Garcés et al. 2020; Graf et al. 2019; Kosch et al. 2019; Tazari et al. 2010) gewonnene Anforderung der modularen und individuellen Unterstützung. Der Fokus liegt dabei auf den nutzerzentrierten Funktionen, um betroffene Personen im Alltag adäquat zu unterstützen. Beispielhaft kann ein ALiS somit differente Funktionen für unterschiedliche Personen bereitstellen, ausgehend von der benötigten Unterstützung. Aufgrund der hohen Individualität der Nutzer*innen sollte bei der Entwicklung eines ALiS speziell der Fokus auf die Erhebung der nutzerzentrierten und individuell bedürfnisorientierten Daten gelegt werden, womit die entsprechende Funktion aus DP3 bereitgestellt werden kann. Im vorliegenden Beitrag wurden die Daten über die beschriebene SLR und MFG erhoben (DF8).

DP4 charakterisiert die Gewährleistung des Datenschutzes, indem DF9 und die damit verbundene lokale Verarbeitung der Daten realisiert werden sollte. Hiermit werden die gewonnenen Informationen (bspw. durch kontextuelle Kameras, Sensoren, etc.) nur für den individuellen Gebrauch genutzt, wodurch die Daten das lokale Umfeld der Nutzer*innen nicht verlassen und somit die notwendige Privatsphäre sichergestellt wird (Garcés et al. 2020; Mueller und Heger 2018; Tazari et al. 2010).

DP5 stellt zugleich die Nutzer*innen in den Mittelpunkt und wird darüber definiert, dass ein ALiS ein service-orientiertes, unterstützendes Angebot darstellt und nicht als auffordernde und nötigende Last empfunden werden sollte. Somit können die Nutzer*innen eine intrinsische Motivation hinsichtlich der Nutzung eine ALiS entwickeln, durch die sie sich autonomer in ihrem häuslichen Umfeld bewegen. Dies wird zudem durch die ethische Betrachtungsweise digitaler Assistenzsysteme bekräftigt, wobei die Faktoren Wohlbefinden, Autonomie und Menschlichkeit implementiert werden (Mueller und Heger 2018). In unserem speziellen Zielgruppenkontext wollen wir dies durch DF1, DF2 und DF 4 erreichen.

Um DR2 zu erfüllen, wird durch DP6 die Nutzung von AR-Technologie ohne tragbare Geräte definiert, wobei gleichzeitig Effizienz und Effektivität adressiert werden. Nutzer*innen sollen somit einen minimalen Aufwand haben, um das System zu bedienen, da sie keinerlei Geräte dafür tragen oder aktiv bedienen müssen. Daraus leiten sich speziell DF10, wodurch möglichst viele Inhalte durch Projektortechnologie dargestellt werden sollen, sowie DF11, welches die Nutzung von Spatial Augmented Reality (Raskar et al. 1998) vorschlägt, ab. Dies umfasst die Nutzung von Projektoren, Kameras, Mikrofonen und Lautsprechern.

Ferner definiert DP7 als Lösungsansatz für DR3 die Nutzung von kontextabhängigen Sensoren (Luís-Ferreira et al. 2019), die nicht für die explizite Kommunikation mit den Nutzer*innen dienen, wodurch die Darstellung kontextabhängiger Informationen intensiviert wird. In Anlehnung an Abb. 3 könnte bspw. über einen Temperatursensor und die Projektion das Szenario Heißer Herd realisiert werden. Dadurch sind beliebig viele Szenarien sowie das Zusammenspiel von unterschiedlichen Sensoren möglich. Im vorliegenden Beitrag wird dies durch DF7 und DF12 die Nutzung der relevanten Technologien weiter spezifiziert.

Abschließend wird durch DP8, welches ebenfalls die Anforderung von DR3 erfüllt, die Integration in bestehende IT-Systeme der Nutzer*innen suggeriert. Durch DF13 wird im vorliegenden Fall die Integration mit individuellen Unterstützungs- 


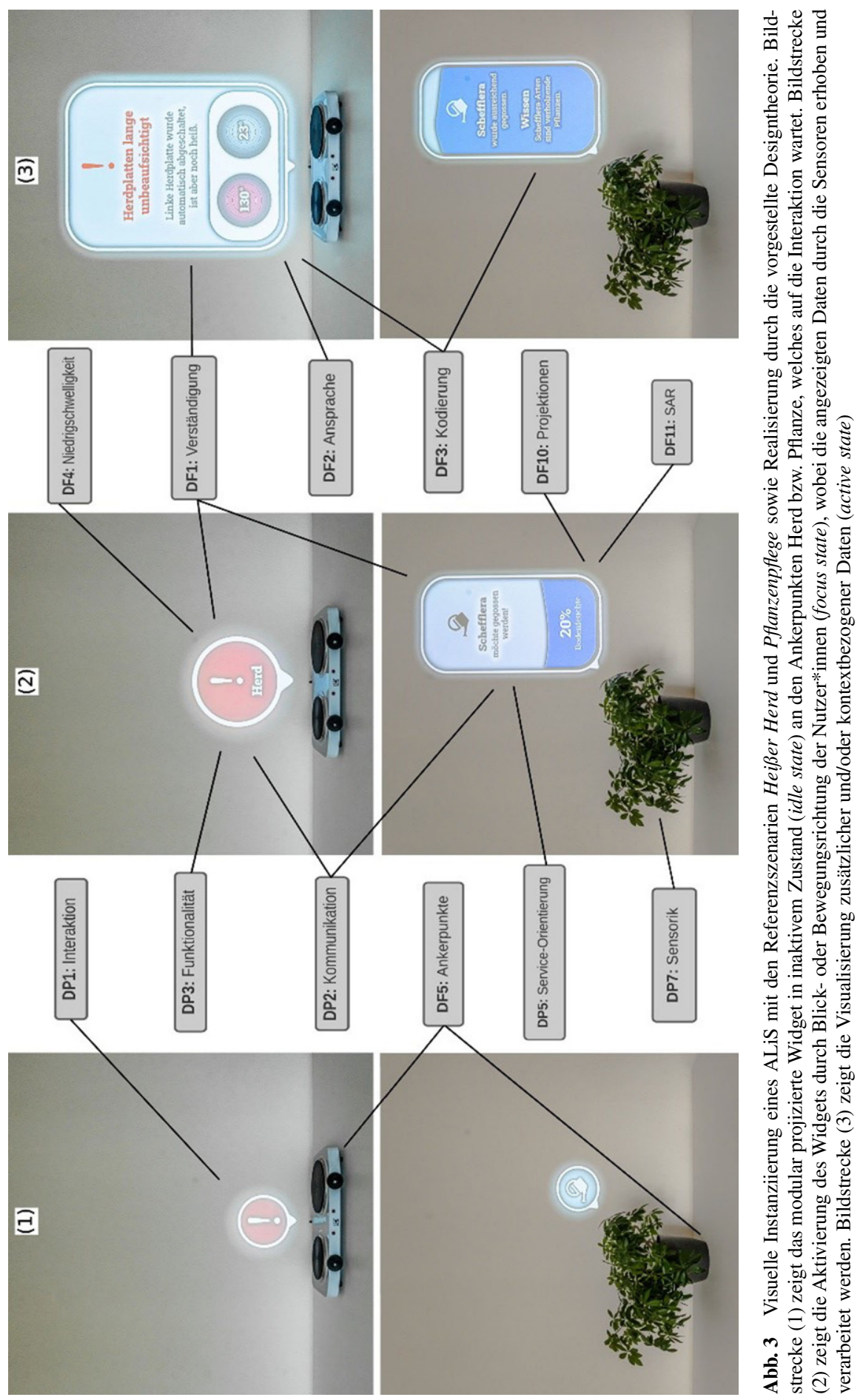


systemen (bspw. Alexa, Digitaler Kalender, Google Home) vorgeschlagen, um Informationen individuell und passgenau auf die jeweiligen Endanwender*innen anzupassen.

Darüber hinaus ermöglicht das Klassifizierungs-Framework von Knote et al. (2019) ALiS als Passive Pervasive Assistant (Typ 4) zu klassifizieren, da das vorliegende digitale Assistenzsystem unaufdringlich sowie suggestiv agiert und in ein digitales Erlebnis mündet. Das System sammelt Daten von verschiedenen Quellen (Sensoren, etc.), erstellt basierend darauf Empfehlungen und beobachtet passiv Kontextzustände (Knote et al. 2019).

\section{Evaluation}

Die Evaluation der entwickelten Designtheorie folgt dem Ansatz von Venable et al. (2017). Hierbei wird großer Wert auf den Nutzen des Artefakts in der Praxis gelegt, einerseits hinsichtlich der Entwicklung kontextabhängiger ALiS-Systeme durch Entwickler*innen, andererseits um die Autonomie älterer sowie kognitiv eingeschränkter Personen zu erhalten oder zu erweitern. Um dies sicherzustellen, werden daher frühe formative Zwischenevaluationen im zugrundeliegenden Forschungsprozess genutzt, um den Fokus in diesem Beitrag auf den praktischen Nutzen der Designtheorie für Forscher*innen zu legen. Der Beitrag konnte dahingehend unsere Forschung theoretisch mit einer zweistufigen SLR und die durchgeführte MFG empirisch stützen. Zur Fortsetzung der Evaluationsstrategie wurde ein Fragebogen entwickelt, der Praktiker*innen und Forscher*innen aus dem allgemeinen IS-Forschungsfeld, ebenso wie aus dem AR-Themengebiet zur wahrgenommenen Nützlichkeit der Designtheorie befragte. Dieser nutzte hierfür die sechs Items der Perceived Usefulness nach Davis (1989), wobei der Fokus der Evaluation auf dem wahrgenommenen Nutzen für Praktiker*innen lag, die anhand der Designtheorie kontextabhängige ALiSSysteme entwickeln wollen. Die sechs Items wurden aus persönlicher Sicht der Proband*innen $(n=10)$ anonymisiert auf einer intervallskalierten, verbal-numerischen 7-Punkt-Likert-Skala ( 1 =Stimme gar nicht zu, ... , 7 = Stimme stark zu) bewertet.

Hinsichtlich der Aussage, dass die Designtheorie es ermöglicht, Aufgaben (bspw. die Entwicklung eines ALiS) schneller und somit effizienter zu erfüllen, zeigten die Teilnehmer*innen eine generelle Zustimmung, wobei die Antworten von starker Zustimmung bis hin zu einer neutralen Bewertung reichten. Hierbei gaben die Proband*innen an, dass die Design Principles dabei helfen würden, schneller die relevanten Bestandteile eines Augmented Living Space zu erkennen und die Bedeutung dieser zu verstehen. Des Weiteren empfinden die Proband*innen die Designtheorie als produktivitäts-steigernd. Drei Personen antworteten dabei mit einer leichten Zustimmung und eine Person mit neutraler Bewertung, wohingegen die restlichen Teilnehmer*innen eine generelle bis starke Zustimmung zeigten. Die anschließende Aussage betraf die persönliche Aufgaben-Performanz und fragte die teilnehmenden Personen, ob die Designtheorie diese im Kontext einer Aufgabe (bspw. Entwickeln eines ALiS) erhöhen würde. Teilnehmer*innen gaben dabei an, dass sie durch die Designtheorie und deren Komponenten schneller Anknüpfungspunkte an bestimmten Design Principles finden würden, um bspw. einzelne Aspekte bei einer Eigenent- 
wicklung zu vertiefen oder anzupassen, was die Produktivität bei der Aufgabenerfüllung erhöhe. Hinsichtlich der Steigerung der individuellen Effektivität durch die Designtheorie, wird diese von den Proband*innen ebenfalls zwischen einer leichten und generellen Zustimmung wahrgenommen, jedoch mit der Tendenz hin zu einer neutralen Bewertung der Aussage. Somit empfinden die Expert*innen, dass ihnen alle relevanten und nützlichen Funktionen zur Aufgabenbewältigung (bspw. Entwicklung eines ALiS) durch die Designtheorie bereitgestellt werden. Daran anknüpfend zeigen die Proband*innen eine tendenziell starke Zustimmung zu der Aussage, dass die Designtheorie die Aufgabenbewältigung vereinfachen würde. Dabei gab es keine Proband*innen, welche die wahrgenommene Nützlichkeit als neutral oder gar ablehnend bewerteten. Letztendlich zeigten alle Expert*innen ihre Zustimmung gegenüber der Aussage, dass sie die Designtheorie hinsichtlich ihrer Aufgabe (bspw. die Entwicklung eines ALiS) als hilfreich ansehen. Dabei gab es nur eine Person, die dies mit schwacher Zustimmung bewertete. Zudem kann der Summenscore der Skalen-Items über alle Fragebögen der Evaluation einen Median von 35 aufweisen, was eine hohe wahrgenommene Nützlichkeit der Designtheorie beschreibt (Skala von 6 bis 42, wobei 6 den niedrigsten Wert und 42 den höchsten zu erreichenden Wert beschreibt). Zusammenfassend zeigt die Auswertung des Fragebogens, dass die Proband*innen die Designtheorie als positiv im Aufgabenkontext empfinden und es eine generelle Zustimmung der Aussagen zur wahrgenommenen Nützlichkeit gibt. Dabei gab es keine Ablehnung der Aussagen und nur wenige neutrale Bewertungen, was die inhaltliche Aussagekraft der DT unterstreicht. Hier konnte die Auswertung zeigen, dass vor allem die Design Requirements Zufriedenheit, Effizienz und Effektivität durch die Design Principles/Design Features erfüllt werden und Praktiker*innen auf diese Weise sinnvolle Anknüpfungspunkte finden.

\section{Schlussfolgerung und nächste Forschungsschritte}

Der vorliegende Beitrag hat gezeigt, dass Spatial Augmented Reality als vielversprechende Lösung zur Unterstützung älterer und kognitiv eingeschränkter Menschen angesehen werden kann. Ziel der zugrundeliegenden Forschung ist dabei die Entwicklung eines SAR-basierten, digitalen Assistenzsystems, um die Autonomie im Alltag betroffener Personen zu erhalten und/oder zu erweitern. Zudem sollen Forscher*innen dabei unterstützt werden, anwendungsspezifische und individuell bedürfnisorientierte ALiS-Systeme zu entwickeln, damit diese in der Praxis zielgruppen-spezifisch eingesetzt werden können. Um die anfänglich formulierte Forschungsfrage dieses Beitrags zu beantworten, wurde ausgehend von einer strukturierten Literaturanalyse und einer moderierten Fokusgruppe eine Designtheorie vorgestellt, deren Quintessenz in einem ersten Szenario und in Hinblick auf unsere kontextabhängige Anwendung dargestellt werden konnte. Abschließend wurde die Designtheorie von Praktiker*innen sowie Forscher*innen evaluiert und erhielt überwiegend positives Feedback, was die praktische Relevanz der DT unterstreicht. Forscher*innen und Praktiker*innen können daher unsere DT adaptieren, um neue und kontextabhängige ALiS-Systeme zu entwickeln. Darüber hinaus tragen die von uns definierten DRs, DPs und DFs, die in einer inter-field DT (Darden und Maull 1977) 
zusammengeführt wurden, zur präskriptiven Wissensbasis der IS-Community nach Gregor und Hevner (2013) sowie zur Wissensbasis des IS-Design-Science-Research nach Woo et al. (2014) bei. Zudem dient die Designtheorie dazu, Forscher*innen zu unterstützen, welche artverwandte ALiS-Systeme für differente Anwendungsszenarien oder Umgebungen entwickeln.

Im Kontext des Nutzens der Designtheorie sollten folgende Limitationen berücksichtigt werden: Einerseits ist die Subjektivität der zugrundeliegenden Design Entscheidungen bei der Entwicklung einer DT eine große Herausforderung. Design Requirements, Design Principles und Design Features unterliegen auch nach rigorosen SLRs oder MFGs der subjektiven Kreativität der forschenden Person, was andererseits im Zweck der Methode begründet liegt. Dabei können andere Forscher*innen selbstredend unterschiedliche Entscheidungen treffen, was in einer abweichenden Designtheorie resultiert. Die konzipierte DT wird darüber hinaus jedoch durch die methodischen Ansätze von Möller et al. (2020) sowie Fu et al. (2016) hinsichtlich der wissenschaftlichen Rigorosität gestützt. Andererseits beschreibt die durchgeführte Evaluation nur eine Stichprobe, womit durch die Wahl anderer Teilnehmer*innen durchaus unterschiedliche Ergebnisse erwartbar wären. Im Kontext zur vorliegenden Forschung sollte daher beachtet werden, dass es sich hierbei nur um eine erste Evaluation der Designtheorie handelte, die zukünftig durch weitere Evaluationen erweitert werden kann. Es kann zudem nicht davon ausgegangen werden, dass die Designtheorie auch tatsächlich alle notwendigen DRs, DPs und DFs für die Entwicklung eines ALiS beinhaltet, was ebenfalls in der zukünftigen Forschung adressiert wird. Dies ermöglicht es, die DT anzupassen, zu präzisieren und bei Bedarf zu vervollständigen.

Mit der vorgestellten Designtheorie dieses Beitrags wird in den nächsten Schritten nun ein Referenzszenario modelliert und entwickelt, welches als Ausgangspunkt für die zukünftige Forschung dient, um eine potenziell angepasste DT vorzustellen, welche speziell durch die Zielgruppe evaluiert werden soll. Zudem sollte der damit verbundene Software- und Hardwareprototyp instanziiert werden. Eine abschließende summative Evaluierung kann außerdem hinsichtlich der drei Komponenten der Gebrauchstauglichkeit durchgeführt werden (Venable et al. 2017; ISO 924111:2018), wobei sich vorbehalten wird, bei Eintritt nicht zufriedenstellender Ergebnisse zusätzliche Forschungszyklen durchzuführen und ALiS somit agil-iterativ weiterzuentwickeln.

Zusatzmaterial online Zusätzliche Informationen sind in der Online-Version dieses Artikels (https://doi. org/10.1365/s40702-021-00830-z) enthalten.

Funding Open Access funding enabled and organized by Projekt DEAL.

Open Access Dieser Artikel wird unter der Creative Commons Namensnennung 4.0 International Lizenz veröffentlicht, welche die Nutzung, Vervielfältigung, Bearbeitung, Verbreitung und Wiedergabe in jeglichem Medium und Format erlaubt, sofern Sie den/die ursprünglichen Autor(en) und die Quelle ordnungsgemäß nennen, einen Link zur Creative Commons Lizenz beifügen und angeben, ob Änderungen vorgenommen wurden.

Die in diesem Artikel enthaltenen Bilder und sonstiges Drittmaterial unterliegen ebenfalls der genannten Creative Commons Lizenz, sofern sich aus der Abbildungslegende nichts anderes ergibt. Sofern das betreffende Material nicht unter der genannten Creative Commons Lizenz steht und die betreffende Handlung 
nicht nach gesetzlichen Vorschriften erlaubt ist, ist für die oben aufgeführten Weiterverwendungen des Materials die Einwilligung des jeweiligen Rechteinhabers einzuholen.

Weitere Details zur Lizenz entnehmen Sie bitte der Lizenzinformation auf http://creativecommons.org/ licenses/by/4.0/deed.de.

\section{Literatur}

Antonino PO, Schneider D, Hofmann C, Nakagawa EY (2011) Evaluation of AAL platforms according to architecture-based quality attributes. In: Keyson D, Maher M, Streitz N, Cheok A, Augusto J, Wichert R, Englebienne G, Aghajan H, Krose B (Hrsg) Ambient intelligence, lecture notes in computer science, Bd. 7040. Springer, Berlin, Heidelberg, S 264-274

Baig MM, Afifi S, Gholam H, Mirza F (2019) A systematic review of wearable sensors and IoT-based monitoring applications for older adults-a focus on ageing population and independent living. J Med Syst 43(8):233. https://doi.org/10.1007/s10916-019-1365-7

Bartsch T, von Arnim C (2019) Kognitive Einschränkungen und Demenzen. In: Maetzler W, Dodel R, Jacobs AH (Hrsg) ICF-basierte Diagnose und Behandlung. Springer, Berlin, S 85-104 https://doi. org/10.1007/978-3-662-57358-7

Baskerville R, Pries-Heje J (2010) Explanatory design theory. Bus Inf Syst Eng 2(5):271-282. https://doi. org/10.1007/s12599-010-0118-4

Baumeister J, Ssin SY, El Sayed NAM, Dorrian J, Webb DP, Walsh JA, Simon TM, Irlitti A, Smith RT, Kohler M, Thomas BH (2017) Cognitive cost of using augmented reality displays. IEEE Trans Visual Comput Graphics 23(11):2378-2388. https://doi.org/10.1109/TVCG.2017.2735098

Benavides A, Gutierrez FJ, Ochoa SF (2020) Hermes: a digital assistant for coordinating invisible work in family elderly caregiving scenarios. In: International conference on human-computer interaction. Springer, Cham, S 437-450

Blackler A, Popovic V (2016) Intuitive interaction research—new directions and possible responses. In: Lloyd P, Bohemia E (Hrsg) Future focused thinking DRS International Conference, Brighton, United Kingdom, 2016, 27 - 30 June. https://doi.org/10.21606/drs.2016.319

Blackler A, Chen LH, Desai S, Astell A (2020) Intuitive interaction framework in user-product interaction for people living with dementia. In: Brankaert R, Kenning G (Hrsg) HCI and design in the context of dementia. Human - computer interaction series. Springer, Cham, S 147-169 https://doi.org/10.1007/ 978-3-030-32835-1_10

Block L, Gilmore-Bykovskyi A, Jolliff A, Mullen S, Werner NE (2020) Exploring dementia family caregivers' everyday use and appraisal of technological supports. Geriatr Nurs 41(6):909-915. https://doi. org/10.1016/j.gerinurse.2020.06.019

Cardoso LF, Mariano FC, Zorzal ER (2020) A survey of industrial augmented reality. Comput Ind Eng. https://doi.org/10.1016/j.cie.2019.106159

Chang YJ, Kang YS, Liu FL (2014) A computer-based interactive game to train persons with cognitive impairments to perform recycling tasks independently. Res Dev Disabil 35(12):3672-3677. https:// doi.org/10.1016/j.ridd.2014.09.009

Chung JC, Man DW (2009) Self-appraised, informant-reported, and objective memory and cognitive function in mild cognitive impairment. Dement Geriatr Cogn Disord 27(2):187-193

Cooper H (1998) Synthesizing research: a guide for literature reviews, 3. Aufl. SAGE, Thousand Oaks

Darbar R, Roo JS, Lainé T, Hachet M (2019) DroneSAR: extending physical spaces in spatial augmented reality using projection on a drone. In: Proceedings of the 18th international conference on mobile and ubiquitous multimedia (MUM '19). Association for Computing Machinery, New York, S 1-7 https://doi.org/10.1145/3365610.3365631

Darden L, Maull N (1977) Interfield theories. Philos Sci 44(1):43-64

Davis FD (1989) Perceived usefulness, perceived ease of use, and user acceptance of information technology. Manag Inf Syst Q 13(3):319-340

Di Donato M, Fiorentino M, Uva AE, Gattullo M, Monno G (2015) Text legibility for projected augmented reality on industrial workbenches. Comput Ind 70:70-78. https://doi.org/10.1016/j.compind.2015.02. 008

Fu KK, Yang MC, Wood KL (2016) Design principles: literature review, analysis, and future directions. J Mech Des. https://doi.org/10.1115/1.4034105 
Garcés L, Oquendo F, Nakagawa EY (2020) Assessment of reference architectures and reference models for ambient assisted living systems: results of a systematic literature review. Int J E-health Med Commun 11(1):17-36

Gauthier S, Reisberg B, Zaudig M et al (2006) International psychogeriatric association expert conference on mild cognitive impairment. Mild cognitive impairment. Lancet. https://doi.org/10.1016/S01406736(06)68542-5

Gomes A, Fernandes K, Wang D (2021) Surface prediction for spatial augmented reality applications. Virtual Real. https://doi.org/10.1007/s10055-020-00490-2

Goonawardene N, Leong C, Tan HP (2018) An action design research of a sensor-based elderly monitoring system for aging-in-place. Thirty Ninth International Conference on Information Systems, San Francisco

Graf R, Benawri P, Whitesall AE, Carichner D, Li Z, Nebeling M, Kim HS (2019) IGYM: an interactive floor projection system for inclusive Exergame environments. In: Proceedings of the annual symposium on computer-human interaction in play (CHI PLAY '19). Association for Computing Machinery, New York, S 31-43 https://doi.org/10.1145/3311350.3347161

Gregor S, Hevner A (2013) Positioning and presenting design science research for maximum impact. Mis Quarterly 37:337-356. https://doi.org/10.25300/MISQ/2013/37.2.01

Grundhöfer A, Iwai D (2018) Recent advances in projection mapping algorithms, hardware and applications. Comput Graph Forum 37:653-675. https://doi.org/10.1111/cgf.13387

Huang B, Sun T, Ling H (2021) End-to-end full projector compensation. IEEE Trans Pattern Anal Mach Intell. https://doi.org/10.1109/TPAMI.2021.3050124

ISO (2018) Ergonomics of human-system interaction-part 11: usability: definitions and concepts (ISO 9241-11:2018(en))

ISO (2019) Ergonomics of human-system interaction-part 210: Human-centered design for interactive systems (ISO 9241-210:2019)

Knote R, Janson A, Söllner M, Leimeister JM (2019) Classifying smart personal assistants: an empirical cluster analysis. In: Proceedings of the 52nd Hawaii international conference on system sciences

Kobayashi D, Hashimoto N (2014) Spatial augmented reality by using depth-based object tracking. In: ACM SIGGRAPH 2014 Posters (SIGGRAPH '14). Association for Computing Machinery, New York https://doi.org/10.1145/2614217.2614226 (Artikel 33)

Kosch T, Wennrich K, Topp D, Muntzinger M, Schmidt A (2019) The digital cooking coach: using visual and auditory in-situ instructions to assist cognitively impaired during cooking. In: Proceedings of the 12th ACM international conference on pervasive technologies related to assistive environments (PETRA '19). Association for Computing Machinery, New York, S 156-163 https://doi.org/10.1145/ 3316782.3321524

Lee YY, Lee JH, Ahmed B, Son MG, Lee KH (2019) A new projection-based exhibition system for a museum. J Comput Cult Herit. https://doi.org/10.1145/3275522

Lema-Sarmiento PA, Calle-Romero PE, Gallegos-Segovia PL, Vintimilla-Tapia PE, Bravo-Torres JF, Ordóñez-Morales EF (2020) SAFER-LIM: a platform for assistance, care and social integration for the elderly. In: International conference on applied technologies. Springer, Cham, S 230-242

Lindlbauer D, Mueller J, Alexa M (2017) Changing the appearance of real-world objects by modifying their surroundings. In: Proceedings of the 2017 CHI conference on human factors in computing systems. Association for Computing Machinery, New York, S 3954-3965 https://doi.org/10.1145/ 3025453.3025795

Lockerbie J, Maiden N (2019) Using a modelling language to describe the quality of life goals of people living with dementia. In: Advanced Information Systems Engineering. CAiSE 2019. Lecture notes in computer science, Bd. 11483. Springer, Cham, S 162-176 https://doi.org/10.1007/978-3-030-212902_11

Luís-Ferreira F, Zamiri M, Sarraipa J, McManus G, O’Brien P, Gonçalves R (2019) Survey on assistive technologies for people with dementia. Ieee Instrum Meas Mag 22(6):45-52. https://doi.org/10.1109/ MIM.2019.8917903

Mayring P, Fenzl T (2019) Qualitative Inhaltsanalyse. In: Baur N, Blasius J (Hrsg) Handbuch Methoden der empirischen Sozialforschung. Springer VS, Wiesbaden https://doi.org/10.1007/978-3-658-21308-4_ 42

Memon M, Wagner SR, Pedersen CF, Beevi FHA, Hansen FO (2014) Ambient assisted living healthcare frameworks, platforms, standards, and quality attributes. Sensors 14(3):4312-4341

Miah SJ, Gammack J, Hasan N (2017) Extending the framework for mobile health information systems research: a content analysis. Inf Syst 69:1-24. https://doi.org/10.1016/j.is.2017.04.001 
Miyamoto J, Koike H, Amano T (2018) Gaze navigation in the real world by changing visual appearance of objects using projector-camera system. In: Proceedings of the 24th ACM symposium on virtual reality software and technology (VRST '18). Association for Computing Machinery, New York, S 1-5 https://doi.org/10.1145/3281505.3281537 (Artikel 14)

Möller F, Guggenberger T, Otto B (2020) Towards a method for design principle development in information systems. 15th International Conference on Design Science Research in Information Systems and Technology, Kristiansand, Norway. https://doi.org/10.1007/978-3-030-64823-7_20

Morgan DL (1997) Focus groups as qualitative research Bd. 2. SAGE, Thousand Oaks https://doi.org/10. $4135 / 9781412984287$

Morris JM (1994) User interface design for older adults. Interact Comput 6(4):373-393. https://doi.org/10. 1016/0953-5438(94)90009-4

Mshali H, Lemlouma T, Moloney M, Magoni D (2018) A survey on health monitoring systems for health smart homes. Int J Ind Ergon 66:26-56. https://doi.org/10.1016/j.ergon.2018.02.002

Mueller M, Heger O (2018) Health at any cost? Investigating ethical dimensions and potential conflicts of an ambulatory therapeutic assistance system through value sensitive design. Thirty Ninth International Conference on Information Systems, San Francisco

Fernando N, Tan FTC, Vasa R, Mouzaki K, Aitken I (2016) Examining digital assisted living. Towards a case study of smart homes for the elderly. In: ECIS 2016: information systems as a global gateway: proceedings of the 24th European conference on information systems. Association for Information Systems, Atlanta, S 1-11

Park SJ, Subramaniyam M, Kim SE, Hong S, Lee JH, Jo CM, Seo Y (2017) Development of the elderly healthcare monitoring system with IoT. In: Advances in human factors and ergonomics in healthcare. Springer, Cham, S 309-315

Picking R, Robinet A, McGinn J, Grout V, Casas R, Blasco R (2012) The Easyline+ project: evaluation of a user interface developed to enhance independent living of elderly and disabled people. Univ Access Inf Soc 11:99-112. https://doi.org/10.1007/s10209-011-0246-8

Preum SM, Munir S, Ma M, Yasar MS, Stone DJ, Williams R, Alemzadeh H, Stankovic JA (2021) A review of cognitive assistants for healthcare: trends, prospects, and future directions. ACM Comput Surv. https://doi.org/10.1145/3419368 (Artikel 130)

Raskar R, Welch G, Fuchs H (1998) Spatially augmented reality. First International Workshop on Augmented Reality, San Francisco

Ro H, Park YJ, Byun JH, Han TD (2019) Display methods of projection augmented reality based on deep learning pose estimation. In: ACM SIGGRAPH 2019 Posters. Association for Computing Machinery, New York, S 1-2 https://doi.org/10.1145/3306214.3338608 (Artikel 55)

Rupprecht P, Kueffner-Mccauley H, Schlund S (2020) Information provision utilizing a dynamic projection system in industrial site assembly. Procedia Cirp 93:1182-1187. https://doi.org/10.1016/j.procir. 2020.04.053

Saborowski M, Kollak I (2015) How do you care for technology? — care professionals' experiences with assistive technology in care of the elderly. Technol Forecast Soc Change 93:133-140. https://doi.org/ 10.1016/j.techfore.2014.05.006

Seet BC, Zhang J, Liu Y (2016) In-home assisted living for elderly dementia care. In: Proceedings of SAI intelligent systems conference. Springer, Cham, S 799-810

Spoladore D, Arlati S, Colombo V, Modoni G, Sacco M (2021) A semantic-enabled smart home for AAL and continuity of care. In: IoT in healthcare and ambient assisted living. Springer, Singapore, S 343-371

Tablado A, Illarramendi A, Bagüés MI, Bermúdez J, Goni A (2005) An intelligent system for assisting elderly people. In: International symposium on methodologies for intelligent systems. Springer, Berlin, Heidelberg, S 466-474

Tavares P, Costa CM, Rocha L, Malaca P, Costa P, Moreira AP, Sousa A, Veiga G (2019) Collaborative welding system using BIM for robotic reprogramming and spatial augmented reality. Autom Constr 106:926-5805. https://doi.org/10.1016/j.autcon.2019.04.020

Tazari MR, Furfari F, Ramos JPL, Ferro E (2010) The PERSONA service platform for AAL spaces. In: Handbook of ambient intelligence and smart environments. Springer, Boston, S 1171-1199

Vaishnavi V, Kuechler W (2015) Design science research methods and patterns: innovating information and communication technology. CRC Press LLC, Boca Raton https://doi.org/10.1201/9781420059335

Varriale L, Briganti P, Mele S (2020) Disability and home automation: insights and challenges within organizational settings. In: Lazazzara A, Ricciardi F, Za S (Hrsg) Exploring digital ecosystems. Lecture notes in information systems and organisation, Bd. 33. Springer, Cham https://doi.org/10.1007/9783-030-23665-6_5 
Venable JR, Pries-Heje J, Baskerville R (2017) Choosing a design science research methodology. https:// aisel.aisnet.org/acis2017/112. Zugegriffen: 21.12.2021, ACIS 2017 Proceedings

Vom Brocke J, Simons A, Niehaves B et al (2009) Reconstructing the giant: on the importance of rigour in documenting the literature search process. https://aisel.aisnet.org/ecis2009/161 (ECIS 2009 Proceedings)

Walls JG, Widmeyer GR, El Sawy OA (1992) Building an information system design theory for vigilant EIS. Inf Syst Res 3(1):36-59

Wegerich A, Dzaack J, Roetting M (2010) Optimizing virtual superimpositions: user-centered design for a UAR supported smart home system. Ifac Proc Vol. https://doi.org/10.3182/20100831-4-FR-2021. 00014 (IFAC-PapersOnline)

Wilson A, Benko H, Izadi S, Hilliges O (2012) Steerable augmented reality with the beamatron. In: Proceedings of the 25th annual ACM symposium on user interface software and technology (UIST '12). Association for Computing Machinery, New York, S $413-422$ https://doi.org/10.1145/2380116. 2380169

Woo C, Saghafi A, Rosales A (2014) What is a contribution to IS design science knowledge? Thirty Fifth International Conference on Information Systems, Auckland

Yang Y, Park YJ, Ro H, Chae S, Han TD (2018) CAre-bot: portable projection-based AR robot for elderly. In: Companion of the 2018 ACM/IEEE international conference on human-robot interaction. Association for Computing Machinery, New York, S 384 https://doi.org/10.1145/3173386.3177528 